\title{
Presence of SERCA and Calcineurin during Fetal Development of Porcine Skeletal Muscle
}

\author{
Henriëtte W. de Jonge, Cornelia W. van der Wiel, Karin Eizema, Wim A. Weijs, and \\ Maria E. Everts \\ Division of Anatomy and Physiology, Department of Pathobiology, Faculty of Veterinary Medicine, Utrecht University, \\ Utretch, The Netherlands
}

\begin{abstract}
SUMMARY Mechanisms involved in skeletal myofiber differentiation during fetal development of large animals are poorly understood. Studies in small animals suggest that the calcineurin $(\mathrm{Cn})$ pathway is involved in myofiber differentiation. Neural activity is a prerequisite for $\mathrm{Cn}$ activity, implying maintenance of sustained low intracellular $\mathrm{Ca}^{2+}$ concentrations. To study the role of $\mathrm{Cn}$ in fetal myofiber differentiation, we monitored the temporal and spatial distribution of $\mathrm{Cn}$ subunits, sarcoplasmic reticulum $\mathrm{Ca}^{2+}$ ATPase (SERCA), phospholamban (PLB), and myosin heavy chain (MyHC) isoforms in relation to ingrowing nerves in porcine semitendinosus muscle ( $\mathrm{m}$. semitendinosus) at 55 and 75 days of gestation ( $\mathrm{dg}$ ) and at term. Immunofluorescence analysis revealed the presence of $\mathrm{Cn}$ subunits and SERCA isoforms at all analyzed stages. Cn distribution was not fiber-type specific, but expression became more prominent at term. At $75 \mathrm{dg}$, differential SERCA2 expression was accompanied by perinuclear PLB in primary fibers. SERCA1 was expressed in all fiber types at all stages. No specific MyHC isoform distribution was seen in relation to neuromuscular contacts, although neuromuscular contacts were present. From these results we speculate that in porcine $\mathrm{m}$. semitendinosus differential SERCA2 expression precedes differential $C n$ expression. The question whether the Cn pathway is involved in prenatal myofiber differentiation needs further studies.
\end{abstract}

(J Histochem Cytochem 54:641-648, 2006)

\author{
KEY WORDS \\ pig \\ myosin heavy chain \\ sarcoplasmic reticulum $\mathrm{Ca}^{2+}$ \\ ATPase \\ phospholamban
}

calcineurin
IN RECENT DECADES, the study of myogenic diversity has been a major issue in muscle developmental biology. It has been demonstrated that myotube development occurs in two or three temporally distinct phases deriving from multiple types of myoblasts (Feldman and Stockdale 1992; Picard et al. 2002). It results in the formation of two or three distinct generations classified as primary, secondary, and tertiary myotubes, according to the type of myosin heavy chains $(\mathrm{MyHC})$ they express. Primary myotubes mature to slow-twitch fibers mostly expressing type I MyHC in the adult. However, in the muscles that become entirely fast they give rise to fast fibers expressing fast $\mathrm{MyHC}$ isoforms. Secondary myotubes often mature to fast fibers in fast

Correspondence to: H.W. de Jonge, Division of Anatomy and Physiology, Department of Pathobiology, Faculty of Veterinary Medicine, Utrecht University, PO Box 80.158, NL-3508 TD, Utrecht, The Netherlands. E-mail: h.w.dejonge@vet.uu.nl

Received for publication August 12, 2005; accepted December 24, 2005 [DOI: 10.1369/jhc.5A6812.2006]. muscles but to fast or slow fibers in mixed muscles (Picard et al. 2002).

In pig, primary myotubes form between 35 and 55 days of gestation $(\mathrm{dg})$, followed by the second generation between 55 and $95 \mathrm{dg}$, and the third generation around birth (Lefaucheur et al. 1995; Picard et al. 2002). During gestation, not only a temporal differentiation takes place but also a spatial differentiation, ultimately leading to a unique architecture. The mature pig skeletal muscle shows a highly organized distribution pattern of fiber types mostly consisting of islets of slow-type I fibers surrounded by an internal ring of fast-type IIA and an external ring of fast-type IIB fibers (Lefaucheur et al. 1995).

The signals and the mechanisms of differentiation playing a role in early myogenesis of the primary fiber generation have recently been elucidated (Buckingham 2001,2003; Christ and Brand-Saberi 2002). Also, the evolution of fiber-type differentiation in developing muscles (of the pig) as well as postnatal muscles has been described (da Costa et al. 2002; Lefaucheur et al. 
2002; Picard et al. 2002). However, the mechanisms underlying the differentiation of the second and third generation of myofibers during fetal development remain poorly understood. For mice, regulation of sarcoplasmic reticulum gene expression during skeletal muscle development has been described (Arai et al. 1992). In addition, the signaling pathway of calcineurin $(\mathrm{Cn})$ has been implicated in controlling slow- vs fast-fiber phenotypes as described for mouse, cell cultures (primary myoblasts, C2C12 myogenic cells), and for fiber-type switching after regeneration in rat (Chin et al. 1998; Friday et al. 2000; Buckingham 2001; McCullagh et al. 2004; Schulz and Yutzey 2004).

Two mechanisms have been implicated in the control of the activity of $\mathrm{Cn}$. First, tonic motor nerve activity, characteristic of nerves innervating slow muscles, has been described to sustain $\left[\mathrm{Ca}^{2+}\right]_{i}$ at levels sufficient to activate $\mathrm{Cn}$ via activation of $\mathrm{Ca}^{2+}$-sensitive calmodulin. It has been shown that $\mathrm{Cn}$ activation selectively establishes the slow muscle phenotype, including expression of slow $\mathrm{MyHC}$ and slow sarcoplasmic reticulum ATP-ase type 2 (SERCA2) (Bigard et al. 2000; Schulz and Yutzey 2004). Second, as Cn is a heterodimeric protein phosphatase composed of a catalytic subunit A and a regulatory subunit B (Rusnak and Mertz 2000), regulation of Cn activity has been proposed through regulation of $\mathrm{CnB}$ protein levels (Mitchell and Pavlath 2002).

A role of the calcineurin pathway in myofiber differentiation during fetal development of large animal muscles has not yet been explored. Myofibers in large mammals such as the pig do not run from tendon to tendon and show multiple innervation zones (Paul 2001). As Cn activation requires electrical nerve activity providing the sustained $\left[\mathrm{Ca}^{2+}\right]_{\mathrm{I}}$, we postulate that this pathway is involved in the achievement of the highly organized patterns of distributed fiber types. We therefore chose to perform this investigation on porcine muscle starting at $55 \mathrm{dg}$ when the formation of the second generation of myofibers had started. Formation of secondary myotubes initiates at the innervation sites of the primary myotubes (Duxson and Sheard 1995), supporting a role of neurogenic control in secondary myotube differentiation. We have chosen the semitendinosus muscle (m. semitendinosus), which has multiple innervation zones and a characteristic rosette myofiber-type distribution. In an earlier study we showed that the porcine $\mathrm{m}$. semitendinosus is already fully innervated at $44 \mathrm{dg}$ when only primary myotubes have developed (De Jonge et al. 2005). From this work, however, we cannot deduce if the neuromuscular contacts are already functional. In the underlying study we have investigated a possible influence of ingrowing nerves and/or neuromuscular contacts on spatial and temporal myofiber differentiation. We monitored at several ages of gestation the distribution of a) expres- sion of MyHC myofiber isoforms and b) the potential regulatory factors of myofiber differentiation: $\mathrm{Cn}$ catalytic unit $\mathrm{A}$ and regulatory unit $\mathrm{B}$, potential regulators of $\left[\mathrm{Ca}^{2+}\right]_{\mathrm{i}}$ SERCA1 and 2 (committed, respectively, to fast- and slow-type myofibers) and phospholamban (PLB, regulator of SERCA2 activity). The distributions are monitored in relation to axonal outgrowth, indicated by the presence of growth-associated protein 43 (GAP43) and neuromuscular contacts, indicated by the presence of acetylcholine receptor (AChR) subunits $\alpha$ and $\gamma$. The $\gamma$ subunit of the ACh receptor is specific for fetal motor nerve endings, whereas the $\alpha$ subunit is present in both pre- and postnatal isoforms of the mammalian receptor (Mishina et al. 1986; Takahashi et al. 2002).

\section{Materials and Methods}

\section{Animals}

Fetal pigs were collected from slaughtered sows (the standard Dutch commercial hybrid race) in a commercial slaughterhouse. Fetal age was estimated from the crown-rump length of the litter, using the growth curves of Evans and Sack (1973). We obtained litters from the prenatal ages of 55 and 75 days postconception, at term, and 112-116 days postconception. Experiments were approved by the Animal Ethical Committee for Animal Experimentation of Utrecht University.

\section{Tissue Collection}

Directly after death of the fetuses, the hind leg attached to the hemisected pelvis was fixed in toto by immersion in phosphate-buffered $4 \%$ formaldehyde for $24 \mathrm{hr}$ at $4 \mathrm{C}$. Dermal incisions of the oldest fetuses increased the exposure to fixation fluid. Thereafter, tissues were stored in $70 \%$ alcohol at $4 \mathrm{C}$ until further preparation.

\section{Vibratome Sections and Free-floating Immunoassay}

The semitendinosus muscle was dissected from the hind legs, embedded in $30 \%$ gelatin, and subsequently cut longitudinally on the vibratome (Micro-cut H1200; Bio-Rad, Hercules, CA) in $80-\mu \mathrm{m}$ sections. For any gestational age, the left and right muscles were taken from two to three fetuses for immunoanalysis. Sections were analyzed by double- or triplefluorescent labeling in free-floating immunoassays. Briefly, sections were rinsed in PBS, permeabilized in $100 \%$ methanol for $10 \mathrm{~min}$ at $-20 \mathrm{C}$, rinsed in PBS, blocked in $0.1 \%$ BSA (fraction $\mathrm{V}, \mathrm{BDH}, \mathrm{UK}$ ) in $\mathrm{PBS}$, and incubated with a mixture of first antibodies in $0.1 \%$ BSA in PBS for $1 \mathrm{hr}$ at room temperature followed by $40 \mathrm{hr}$ at $4 \mathrm{C}$. The sections were then rinsed in large volumes of PBS, followed by the incubation with the mixture of secondary fluorescent conjugated antibodies in $0.1 \%$ BSA in PBS for $1.5 \mathrm{hr}$ at room temperature. Finally, the sections were rinsed in large volumes of PBS, followed with a quick rinse in $\mathrm{dH}_{2} \mathrm{O}$, mounted in Fluorsave Reagent (Calbiochem; EMD Biosciences, Inc., Darmstadt, Germany) on SuperFrost Plus (Menzel-Gläzer; Braunschweig, Germany) glass slides and left to dry at 37C. 
To control the performance of the resulting assay, for example, in background staining in every free-floating immunoassay, all incubations were paralleled by negative controls treated similarly with the exception of the first antibody.

\section{Antibodies}

Sections were stained using the following first antibodies: antiskeletal muscle slow MyHC (NOQ7.5.4D), mouse monoclonal $\operatorname{IgG}$, dilution 1:2000 and anti-skeletal fast MyHC (MY-32), mouse monoclonal IgG, dilution 1:2000 (both from Sigma-Aldrich; St Louis, MO); anti-SERCA1 ATPase (IIH11), mouse monoclonal IgG, dilution 1:500, and anti-SERCA2 ATPase (IID8), mouse monoclonal IgG, dilution 1:500 (both from Abcam; Cambridge, UK); anti-calcineurin A (PP2B-A, clone H-209), rabbit polyclonal IgG, dilution 1:100 (Santa Cruz Biotechnology, Santa Cruz, CA), anti-calcineurin B, rabbit polyclonal, dilution 1:100 (Affinity BioReagents; Golden, $\mathrm{CO})$; anti-AChR $\alpha 2$ (H-111), rabbit polyclonal $\mathrm{IgG}$, dilution 1:100 and anti-AChR $\gamma$ (H-172), rabbit polyclonal IgG, dilution 1:100 (both from Santa Cruz Biotechnology; tebubio b.v., The Netherlands); anti-GAP43 (H-100), rabbit polyclonal IgG, dilution 1:100 (Santa Cruz Biotechnology). Anti-phospholamban (clone A1), mouse monoclonal IgG, dilution 1:100, was purchased from Upstate (Lake Placid, NY). Secondary monoclonal antibodies used were goat antimouse or goat anti-rabbit, highly cross-adsorbed whole antibody conjugates Alexa Fluor 488 or 568 at a dilution of 1:100, and nuclear staining was performed with TO-PRO-3 iodide, all purchased from Molecular Probes (Eugene, OR).

\section{Analysis}

Vibratome sections were analyzed on a Leica TCS Sp (Wetzlar, Germany) confocal laser scanning microscope using laser lines 488, 568, and $633 \mathrm{~nm}$, and images shown are composed of these sequential scannings. Negative controls were set on the background settings of the positive controls taken on the confocal scanning microscope.

\section{Results}

\section{MyHC Expression and Neuromuscular Contacts}

We monitored the spatial distribution of neuronspecific proteins in relation to the fiber-type pattern in the porcine fetal $\mathrm{m}$. semitendinosus. To study the presence of the neuromuscular junctions, antibodies against ACh-receptor subunits $\alpha$ and $\gamma$ were used. Figure 1 illustrates the expression pattern of ACh-receptor subunit $\alpha$ (Figures 1A-1C and $\gamma$ (Figures 1D-1F) in combination with the expression of slow MyHC (Figures 1A,1C-1F) or fast $\mathrm{MyHC}$ (Figure 1B) at three different stages of development. Both receptor type units were already present at $55 \mathrm{dg}$.

The presence of positive staining for GAP43 at all presented gestation ages (Figures 1G-1I) confirmed our earlier findings that the porcine $\mathrm{m}$. semitendinosus was already fully innervated as early as $44 \mathrm{dg}$ with a finemeshed neural network supplying the whole muscle (De Jonge et al. 2005). In the latter study we applied the classical silver staining method to visualize the ingrowing fetal nerves, which revealed the same patterns found by immunohistochemistry presented in the present study. In addition, staining fetal $\mathrm{m}$. semitendinosus with Alexa 488-conjugated $\alpha$-bungarotoxin presented similar patterns (data not shown). Figures 1G-1I illustrate the combination of GAP 43 with the expression of slow MyHC (Figures $1 \mathrm{H}$ and $1 \mathrm{I}$ ) and fast MyHC (Figures $1 \mathrm{~B}$ and $1 \mathrm{G}$ ). We could not reveal a specific expression pattern directly related to the presence of neuromuscular junctions or ingrowing nerves at any of the analyzed gestational ages.

\section{Expression of SERCA and PLB}

Activity of the calcineurin pathway is determined by $\left[\mathrm{Ca}^{2+}\right]_{\mathrm{I}}$, which in turn is controlled by many proteins located in the sarcoplasmic membrane. One of these proteins is PLB-regulating SERCA. Differences in expression patterns of SERCA1 and 2 and PLB were found (Figure 2 and Figure 3). Because antibodies used against slow-type MyHC proteins and SERCA were both raised in mouse, we tried to use another antibody against slow $\mathrm{MyHC}$, also raised in mouse but of type IgM (A4.840; Alexis Biochemicals, Lausen, Switzerland). Unfortunately, this antibody did not give any positive staining, making it impossible to achieve double staining of slow MyHC and SERCA. Using CnA (red) as a counterstaining, we could detect SERCA1 expressed in sarcomeric banding patterns in all fibers at 55 and $75 \mathrm{dg}$ (Figures 2A and 2B). At term (Figure 2C), SERCA1 is still expressed at the sarcomeres as well as in clusters throughout all myofibers, as can clearly be observed in Figures 3A and 3B. SERCA2 expression was irregular at $55 \mathrm{dg}$ (Figure 2D). At $75 \mathrm{dg}$ it was restricted to large fibers, assumed to be primary fibers, in banding patterns but also concentrated around the nucleus (Figure 2E). At term, SERCA2 expression was located near the sarcomeres of large, central fibers (Figure 2F). In Figures 3D-3F, cross-sections of m. semitendinosus at term are presented, clearly showing the expression of SERCA2 in central fibers. The expression of the SERCA2 regulator PLB was similar to SERCA2 expressed in the large central fibers. This was first seen at $75 \mathrm{dg}$ (Figure 2H). Distribution of PLB within each fiber was remarkable. As revealed by the use of a nuclear staining with TO-PRO-3 iodide (Figures $2 \mathrm{H}^{*}$ and $\left.2 \mathrm{I}^{*}\right)$, it was solely concentrated at the periphery of the nucleus. At 55 dg, PLB expression was undetectable.

\section{Calcineurin Expression}

We have analyzed the expression of both $\mathrm{CnA}$ and $\mathrm{B}$ at different gestational ages. Both subunits were expressed in all fibers at all ages (Figures 4A-4F), and both subunits became more prominent at term (Figures $4 \mathrm{C}$ and $4 \mathrm{~F}$ ). At $55 \mathrm{dg}, \mathrm{CnA}$ expression is irregular (Figure $2 \mathrm{G}$ 

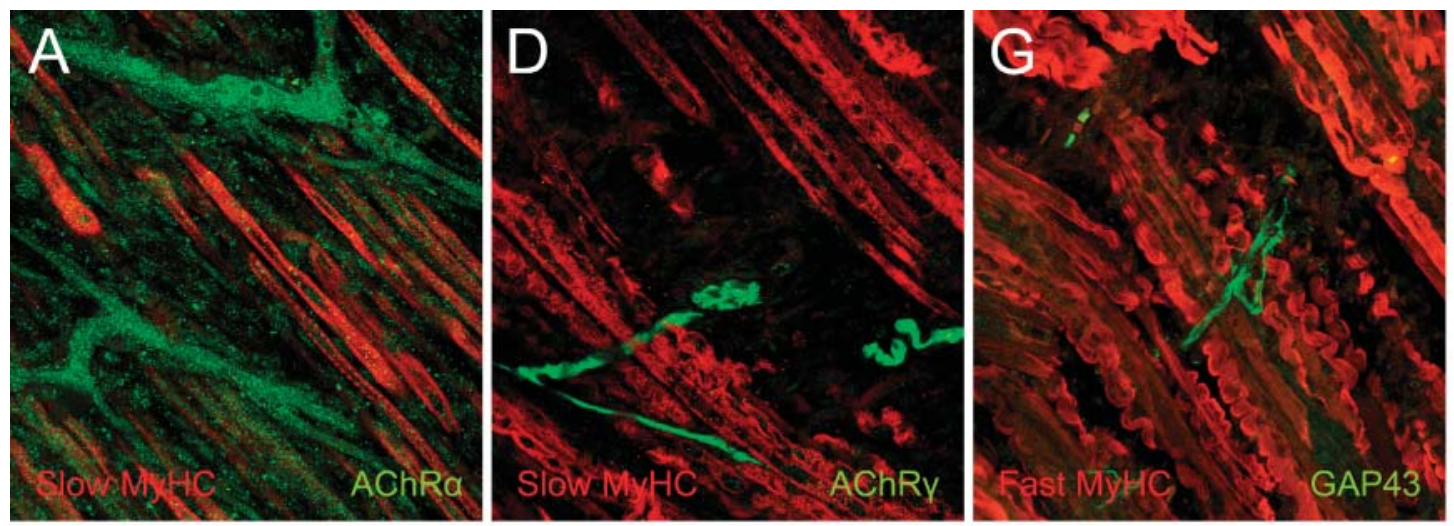

$55 \mathrm{dg}$
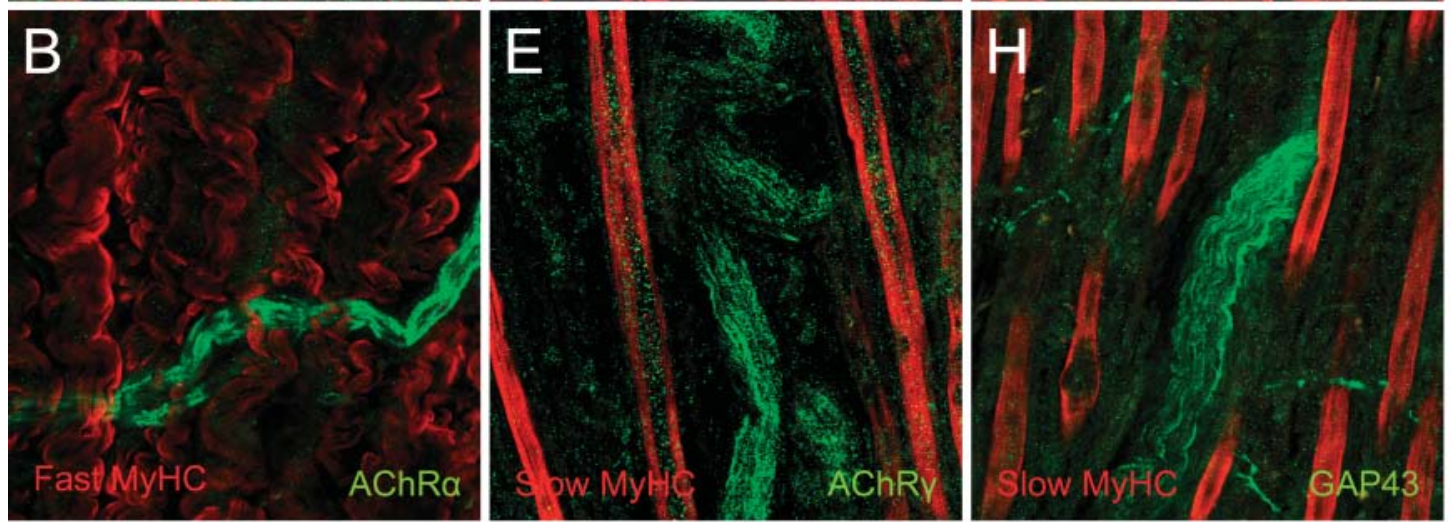

$75 \mathrm{dg}$
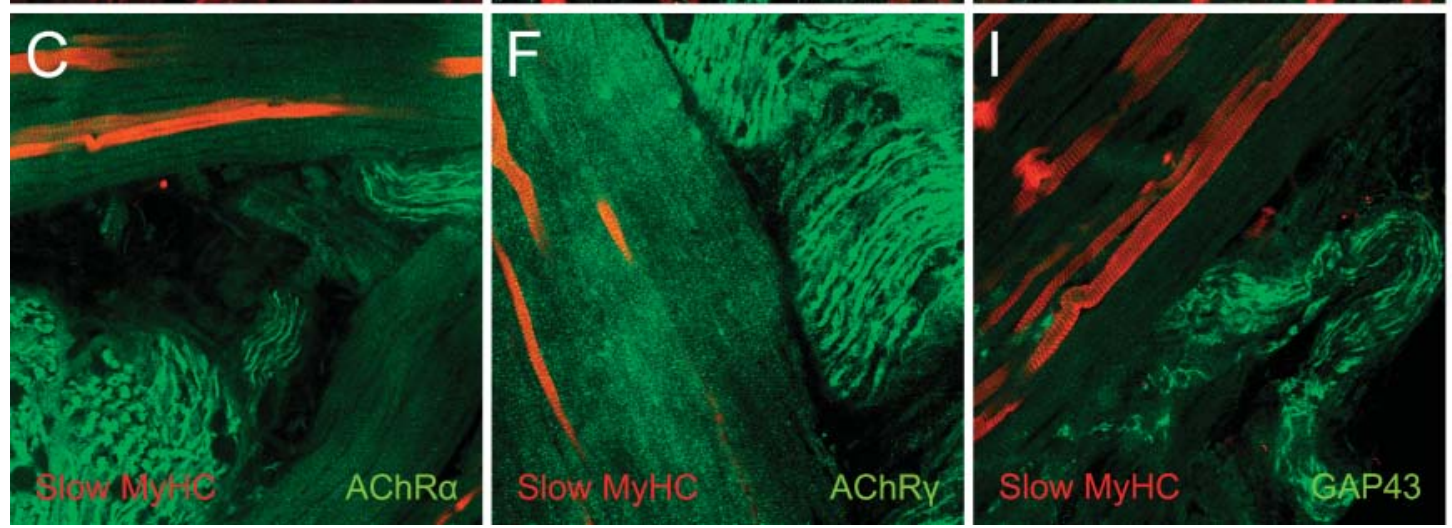

At term

Figure 1 Immunoanalysis of the expression of myosin heavy chain (MyHC) isoforms slow or fast in relation to the presence of acetylcholine receptor (AchR) subunits $\alpha$ or $\gamma$ or growth-associated protein 43 (GAP43) at 55 or $75 \mathrm{dg}$ or at term in the porcine semitendinosus muscle (m. semitendinosus). In green: AChR subunits $\alpha(\mathbf{A}-\mathbf{C})$ or $\gamma(\mathbf{D}-\mathbf{F})$ or GAP43 (G-I). In red: MyHC isoforms slow $(\mathbf{A}, \mathbf{C}-\mathbf{F}, \mathbf{H}, \mathbf{I})$ or fast $(\mathbf{B}, \mathbf{G})$ at 55 dg $(\mathbf{A}, \mathbf{D}, \mathbf{G})$ and $75 \mathrm{dg}(\mathrm{B}, \mathrm{E}, \mathrm{H})$ or at term $(\mathbf{C}, \mathrm{F}, \mathrm{I})$. Magnification: $\times 400$.

and Figure 3A), whereas at $75 \mathrm{dg}$ it is more ubiquitous; at term, expression of $\mathrm{CnA}$ is found to be in small concentrates clearly seen in Figure 2I and Figures 3A, $3 \mathrm{C}, 3 \mathrm{D}$, and $3 \mathrm{~F}$. It is, however, difficult to discern whether it is colocalized or localized in between sarcomeric proteins (Figure $4 \mathrm{C}$ ). $\mathrm{CnB}$ at $55 \mathrm{dg}$ is expressed more diffusely, although the image obtained with the 488-nm laser line revealed expression near the sarcomeres (Figure 4D). This diffuse expression of $\mathrm{CnB}$ was also seen at $75 \mathrm{dg}$ (Figure 4E), whereas at term $\mathrm{CnB}$ expression seemed to reveal a banding pattern (Figure 4F).

\section{Discussion}

The general process of myogenesis is similar in different species. However, the kinetics of muscle fiber development appears to be different among species depending on their maturity at birth (Picard et al. 2002). For small laboratory animals it has been 

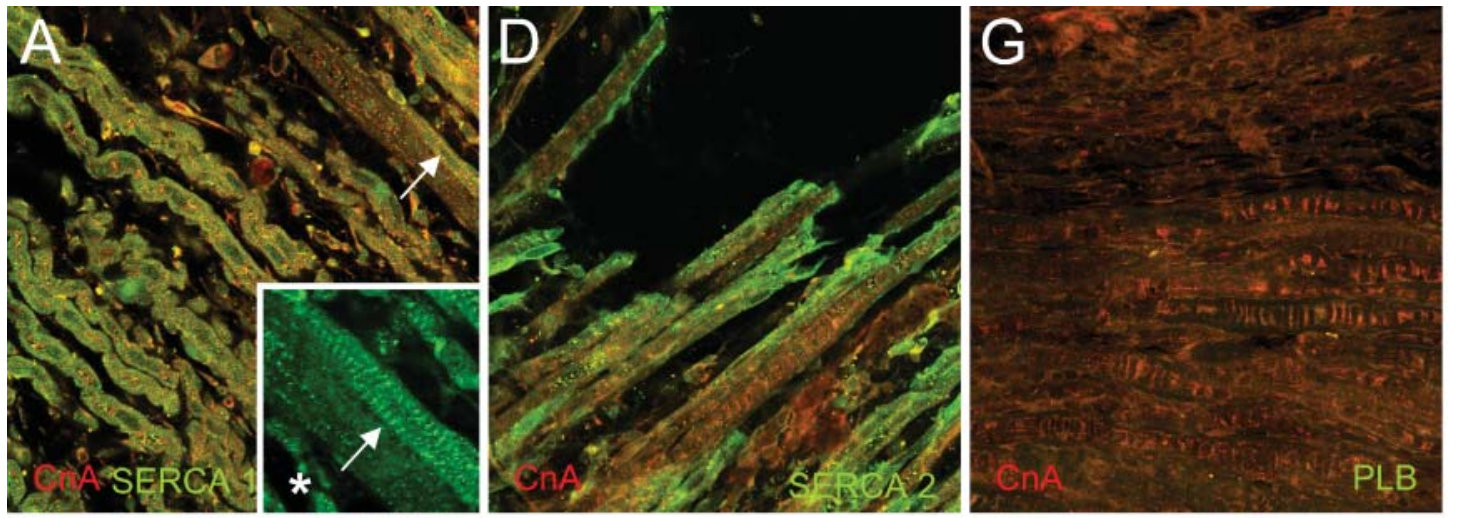

$55 \mathrm{dg}$
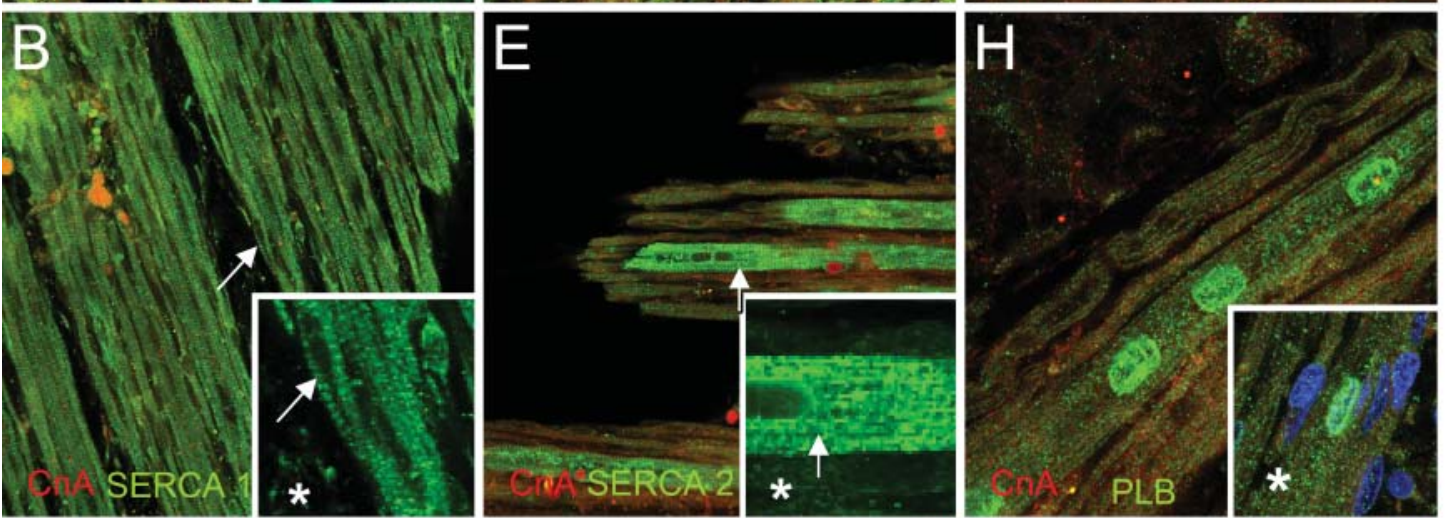

$75 \mathrm{dg}$
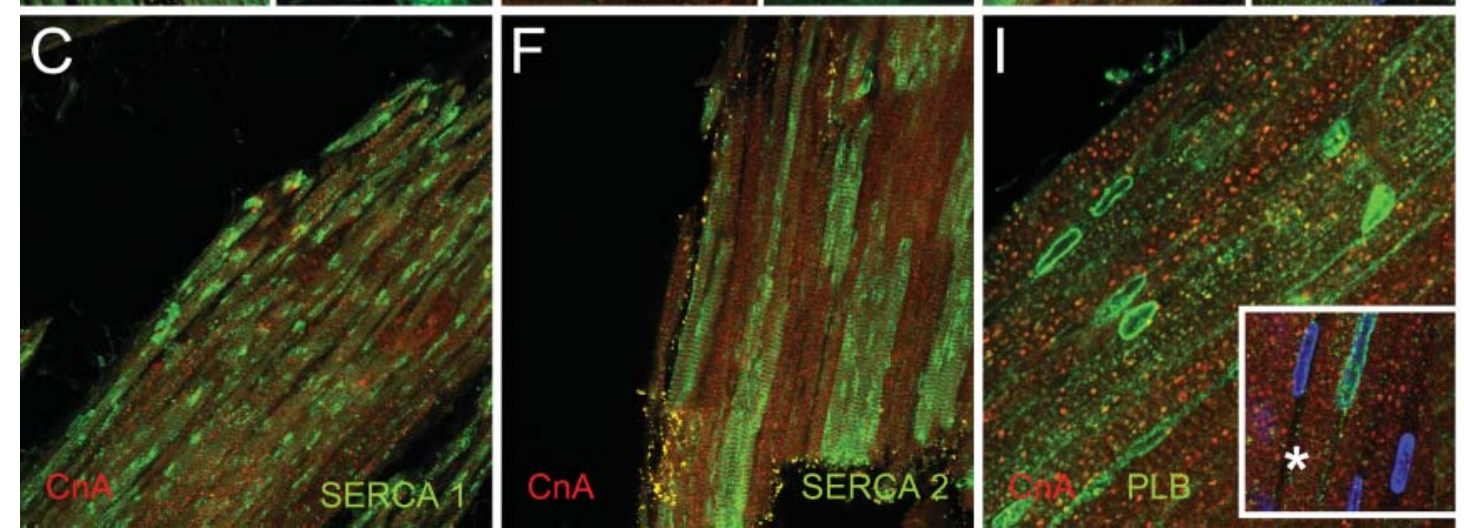

At term

Figure 2 Immunoanalysis of the expression of sarcoplasmic reticulum $\mathrm{Ca}^{2+}$ ATPase 1(SERCA1), SERCA2, or phospholamban (PLB) in the porcine $\mathrm{m}$. semitendinosus at 55 or $75 \mathrm{dg}$ or at term. In green: SERCA1 at $55 \mathrm{dg}(\mathrm{A}), 75 \mathrm{dg}$ (B), and at term (C), SERCA2 at $55 \mathrm{dg}$ (D), $75 \mathrm{dg}$ (E), and at term $(\mathbf{F})$, and PLB at $55(\mathbf{G}), 75(\mathbf{H}) \mathrm{dg}$, and at term (I). In red: calcineurin $A(C n A)$. At the inset $\left(\mathbf{A}^{*}, \mathbf{B}^{*}, \mathrm{E}^{*}\right)$ a detail is shown of the structures (white arrows, $A, B, E)$, respectively; for more clarity these are given for the separate green fluorescent signal. At the inset $\left(H^{*}, I^{*}\right)$ nuclear staining with TO-PRO-3 iodide (blue) is combined with PLB staining (green) to show that PLB is specifically expressed at nuclei. Magnification: A-G = $\times 400$; $H, H^{*}, \mathbf{l}, I^{*}=\times 1000$.

described that skeletal muscles are highly undifferentiated at birth and then undergo a marked process of differentiation in attaining the adult phenotype (Baldwin and Haddad 2001), whereas fiber-type differentiation in large farm-animal species has its onset before birth (Picard et al. 2002). The phenotypic differentiation leading to the mature fiber identity is influenced by innervation. The neural initiation site on the primary myofibers correlates with the onset of secondary myofiber for- mation (Gunning and Hardeman 1991; Zhang and McLennan 1998).

\section{MyHC Expression}

In large animals such as the pig, results on myofiber typing of fetal and adult muscle based on histochemistry and immunohistochemistry using non-commercial antibodies may differ (Lefaucheur et al. 2002) due to 

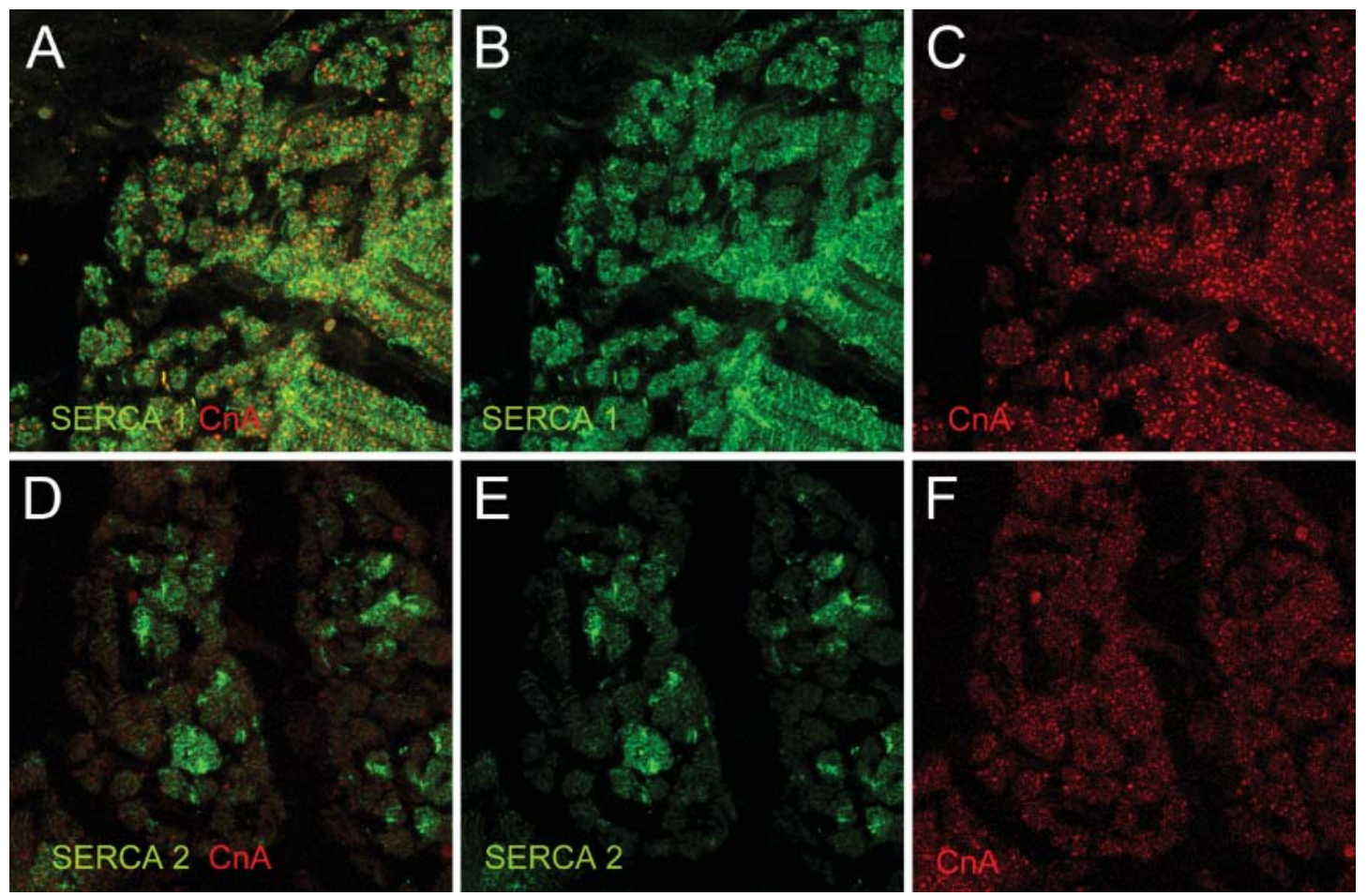

Figure 3 Immunoanalysis of the expression of SERCA1 (green, A-C) and SERCA2 (D-F, in green) in combination with CnA expression (in red) in cross-sections of the porcine $m$. semitendinosus at term. For more clarity, separate images of the green $(\mathbf{B}, \mathbf{E})$ and red $(\mathbf{C}, \mathbf{F})$ signals are presented. Magnification: $\times 400$.

the lack of reliable antibodies specific for pig, as well as the presence of a high proportion of hybrid fibers (Quiroz-Rothe and Rivero 2004; Toniolo et al. 2004). Lack of specific antibodies against fast $\mathrm{MyHC}$ isoforms seriously hampered our study. Although we did not find clear differences in distribution of slow or fast $\mathrm{MyHC}$ in the area of neuromuscular contacts, we cannot rule out a specific distribution of the several fast $\mathrm{MyHC}$ isoforms. Because we were interested in the spatial distribution of myofiber types and other factors, we used relatively thick formalin-fixed vibratome sections and free-floating immunoassays, further complicating specific antibody binding.

\section{SERCA and PLB Expression}

In fetal porcine $\mathrm{m}$. semitendinosus, SERCA1 appears in all fiber types as seen in the cross-sections at term, although a reduced expression at primary fibers cannot be ruled out with certainty because of the impossibility of double staining with anti-type-I antibody. At term, SERCA1 expression in condensates, as well as in a banding pattern near the sarcomeres, may imply reorganization in quantity or localization of protein expression. Expression of SERCA2 in conjunction with PLB in assumed primary fibers is supported by the study of Quiroz-Rothe and Rivero (2004). These authors found a nearly unique correspondence in the distribution of sarcoplasmic reticulum-related proteins, such as slow isoforms of SERCA and slow MyHC, PLB, and slow $\mathrm{MyHC}$ but also fast MyHC and fast SERCA isoforms in adult porcine $\mathrm{m}$. longissimus lumborum, which is similar to findings reported for fetal mice (Arai et al. 1992). In fetal porcine m. semitendinosus, PLB at $75 \mathrm{dg}$ was convincingly found to be sublocalized at the periphery of the nucleus possibly colocalized with SERCA2 as also SERCA2 density was increased around the nucleus (Figures 2E and 2H). This peculiar expression pattern was consistent and was found in several specimens at $75 \mathrm{dg}$, as well as at term. Recently, it has become clear that nuclear calcium signals are also regulated at the nuclear envelope and nuclear reticulum (Echevarria et al. 2003) by, e.g., SERCA-type Ca-ATPase (Bachs et al. 1994; Zhao et al. 2004). Because it is generally accepted that PLB is coexpressed with the SERCA2 gene in slow-twitch skeletal muscle, PLB may be (temporally) expressed in the nuclear envelope in a similar manner, e.g., X,K-ATPase $\beta$-subunit of which a unique temporospatial distribution in the nuclear envelopes of perinatal myocytes has been described (Zhao et al. 2004).

\section{Calcineurin Expression}

In rabbit soleus muscle, $\mathrm{CnB}$ was immunocytochemically found to be expressed in a diffuse way in both fast and slow fiber types and to be arranged in cross-striated 
Figure 4 Immunoanalysis of the expression of subunits $\mathrm{CnA}$ and $\mathrm{CnB}$ in the porcine $\mathrm{m}$. semitendinosus at $55 \mathrm{dg}$, $75 \mathrm{dg}$, or at term. In green: $\mathrm{CnA}$ at $55 \mathrm{dg}$ (A), $75 \mathrm{dg}$ (B), and at term (C), CnB at $55 \mathrm{dg}(\mathrm{D}), 75 \mathrm{dg}(\mathrm{E})$, and at term (F). In red: slow MyHC. (C*) Example of another orientation of myofibers of the same specimen showing yellow staining indicating $\mathrm{CnA}$ and slow $\mathrm{MyHC}$ expression in the same fiber. $\left(D^{*}, F^{*}\right) A$ detail of the structures indicated by the white arrows in $\mathbf{D}$ and $\mathbf{F}$, respectively. $\mathrm{CnB}$ subunit is expressed in a banding pattern. Magnification: $\times \mathbf{4 0 0}$
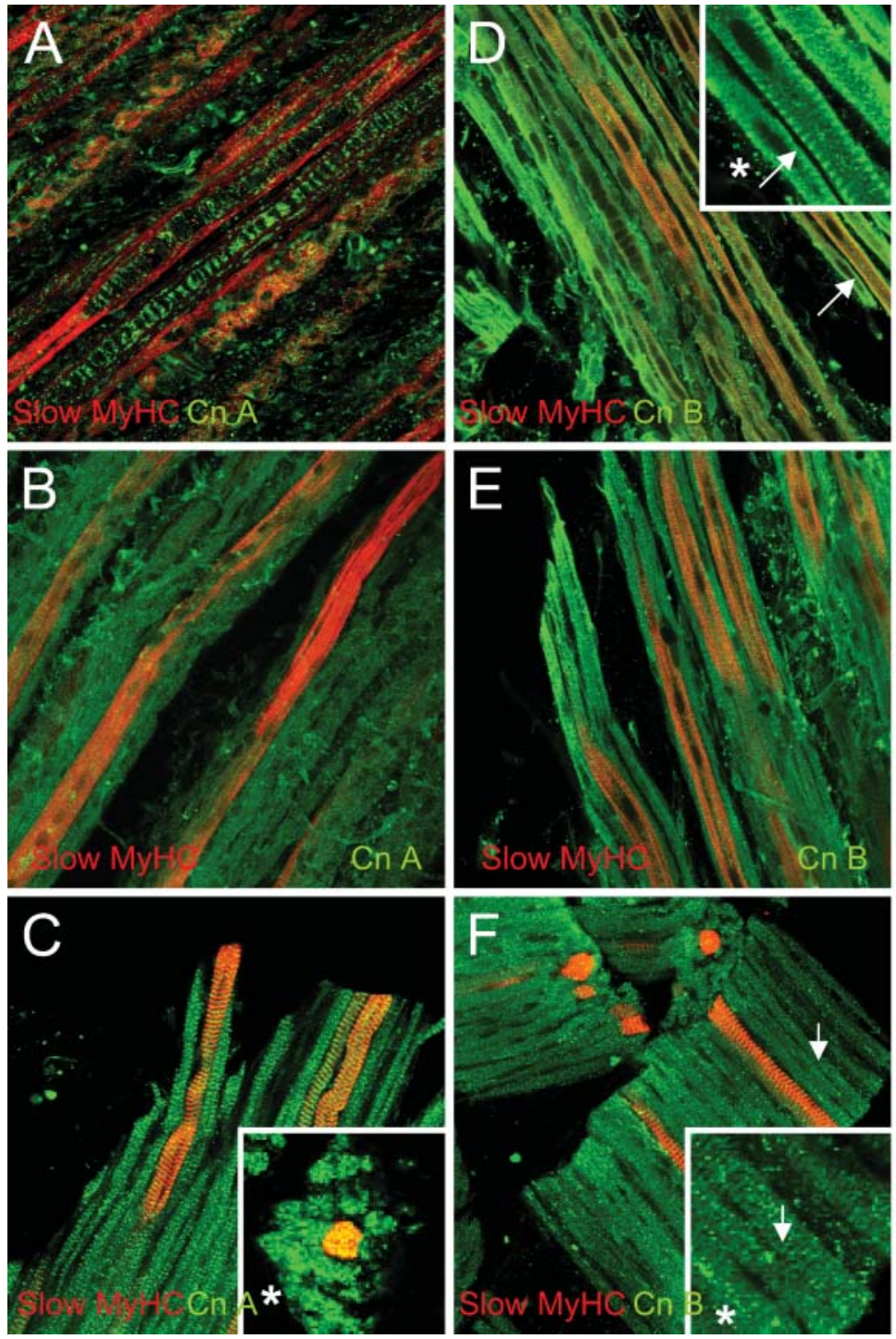

\section{$55 \mathrm{dg}$}

$75 \mathrm{dg}$

\section{At term}

bands in functioning muscles (Sacchetto et al. 2001). Our findings on $\mathrm{Cn}$ subunit distribution in porcine $\mathrm{m}$. semitendinosus corroborate these findings. In developing muscle, $\mathrm{CnB}$ revealed a similar pattern of diffused expression but in a cross-striated pattern at term. CnA expression pattern in concentrates, as we found in fetal porcine muscle, was not described as such by Sacchetto et al. (2001). However, Torgan and Daniels (2006) showed a similar expression pattern in cultured rat myotubes. They showed that calcineurin staining became more prominent in mature myotubes and that it began to exhibit a cytoplasmic pattern that included rows of punctua to the developing myofibrils, supposedly on either side of the Z-line in a pattern consistent with that of triads (Torgan and Daniels 2006). Differences in expression pattern or quantity may serve a regulatory role. An unequal distribution of the $\mathrm{Cn}$ subunits over different muscles leads to differences in ac- tivity levels, as has been described for the plantaris and soleus muscle in mouse (Mitchell et al. 2002).

In developing porcine $\mathrm{m}$. semitendinosus, distribution of the $\mathrm{Cn}$ subunits is not associated with the spatial or temporal distribution of the $\left[\mathrm{Ca}^{2+}\right]_{i}$ regulator SERCA. In the pig, the temporal distribution of SERCA runs, in turn, parallel to myofiber type differentiation, e.g., concerning $\mathrm{MyHC}$ isoform expression, which starts at $\sim 70-75$ dg (Picard et al. 2002). Cn activity is not only responsible for myofiber differentiation but is also implicated in maintenance of muscle calcium homeostasis (Mitchell et al. 2002), which may be the prenatal role for $\mathrm{Cn}$. The maintenance of the appropriate $\left[\mathrm{Ca}^{2+}\right]_{\mathrm{i}}$ in slow fibers by SERCA2 and PLB may, therefore, be a prerequisite for maintenance of the slow (primary) fiber type. According to the fiber-type differentiation evolution scheme given by Picard et al. (2002), although starting at $70-75 \mathrm{dg}$, the major differentiation of porcine sec- 
ondary fibers occurs postnatally. As the kinetics of myofiber development is dependent on the maturity at birth, we may be able to explain our results in pig based on our laboratory findings in horse. Elevated levels of $\mathrm{CnA}$ and $\mathrm{CnB}$ were associated with SERCA2 expression in slow $\mathrm{MyHC}$ fibers in the equine $\mathrm{m}$. gluteus medius at 28 days before birth (Eizema K, unpublished data). In pig, at birth less mature than horse, we found that $\mathrm{CnA}$ is clearly present, and $\mathrm{CnB}$ reveals a banding pattern. However, the distribution is not slow-fiber-type specific. A contribution of $\mathrm{Cn}$ in porcine secondary myofiber differentiation might be postponed and occur postnatally.

In summary, to study fetal myofiber differentiation, we have analyzed the temporal and spatial distribution of MyHC isoforms, Cn, and SERCA $1 / 2$ and PLB in developing porcine $\mathrm{m}$. semitendinosus in relation to neuromuscular contacts. Although we could not distinguish a specific $\mathrm{MyHC}$ isoform distribution, from $75 \mathrm{dg}$ onward, SERCA2 was specifically expressed in large, presumably primary fibers, concomitant with a peculiar but consistent expression of PLB. SERCA1 was expressed in all fibers. Cn subunit expression was analyzed, and both subunits appeared more prominent at term. We speculate that in correspondence to evolution of porcine myofiber differentiation, in the m. semitendinosus, calcineurin may be involved in myofiber differentiation after birth.

\section{Acknowledgments}

We thank Ing. Anko de Graaff and Dr. Richard Wubbolts of the Center for Cell Imaging (Faculty of Veterinary Medicine, Utrecht University) for technical advice.

\section{Literature Cited}

Arai M, Otsu K, MacLennan DH, Periasamy M (1992) Regulation of sarcoplasmic reticulum gene expression during cardiac and skeletal muscle development. Am J Physiol 262:C614-620

Bachs O, Agell N, Carafoli E (1994) Calmodulin and calmodulinbinding proteins in the nucleus. Cell Calcium 16:289-296

Baldwin KM, Haddad F (2001) Effects of different activity and inactivity paradigms on myosin heavy chain gene expression in striated muscle. J Appl Physiol 90:345-357

Bigard X, Sanchez H, Zoll J, Mateo P, Rousseau V, Veksler V, Ventura-Clapier R (2000) Calcineurin co-regulates contractile and metabolic components of slow muscle phenotype. J Biol Chem 275:19653-19660

Buckingham M (2001) Skeletal muscle formation in vertebrates. Curr Opin Genet Dev 11:440-448

Buckingham M (2003) How the community effect orchestrates muscle differentiation. Bioessays 25:13-16

Chin ER, Olson EN, Richardson JA, Yang Q, Humphries C, Shelton $\mathrm{JM}, \mathrm{Wu} \mathrm{H}$, et al. (1998) A calcineurin-dependent transcriptional pathway controls skeletal muscle fiber type. Genes Dev 12: 2499-2509

Christ B, Brand-Saberi B (2002) Limb muscle development. Int J Dev Biol 46:905-914

da Costa N, Blackley R, Alzuherri H, Chang KC (2002) Quantifying the temporospatial expression of postnatal porcine skeletal myosin heavy chain genes. J Histochem Cytochem 50:353-364

De Jonge HW, De Bakker MA, Verbeek FJ, Weijs WA (2005) Em- bedding of large specimens in glycol methacrylate: prerequisites for multi-signal detection and high-resolution imaging. Microsc Res Tech 66:25-30

Duxson MJ, Sheard PW (1995) Formation of new myotubes occurs exclusively at the multiple innervation zones of an embryonic large muscle. Dev Dyn 204:391-405

Echevarria W, Leite MF, Guerra MT, Zipfel WR, Nathanson MH (2003) Regulation of calcium signals in the nucleus by a nucleoplasmic reticulum. Nat Cell Biol 5:440-446

Evans HE, Sack WO (1973) Prenatal development of domestic and laboratory mammals: growth curves, external features and selected references. Zentralbl Veterinarmed [C] 2:11-45

Feldman JL, Stockdale FE (1992) Temporal appearance of satellite cells during myogenesis. Dev Biol 153:217-226

Friday BB, Horsley V, Pavlath GK (2000) Calcineurin activity is required for the initiation of skeletal muscle differentiation. J Cell Biol 149:657-666

Gunning P, Hardeman E (1991) Multiple mechanisms regulate muscle fiber diversity. FASEB J 5:3064-3070

Lefaucheur L, Ecolan P, Plantard L, Gueguen N (2002) New insights into muscle fiber types in the pig. J Histochem Cytochem 50: 719-730

Lefaucheur L, Edom F, Ecolan P, Butler-Browne GS (1995) Pattern of muscle fiber type formation in the pig. Dev Dyn 203:27-41

McCullagh KJ, Calabria E, Pallafacchina G, Ciciliot S, Serrano AL, Argentini C, Kalhovde JM, et al. (2004) NFAT is a nerve activity sensor in skeletal muscle and controls activity-dependent myosin switching. Proc Natl Acad Sci USA 101:10590-10595

Mishina M, Takai T, Imoto K, Noda M, Takahashi T, Numa S, Methfessel C, et al. (1986) Molecular distinction between fetal and adult forms of muscle acetylcholine receptor. Nature 321:406-411

Mitchell PO, Mills ST, Pavlath GK (2002) Calcineurin differentially regulates maintenance and growth of phenotypically distinct muscles. Am J Physiol Cell Physiol 282:C984-992

Mitchell PO, Pavlath GK (2002) Multiple roles of calcineurin in skeletal muscle growth. Clin Orthop 403:S197-202

Paul AC (2001) Muscle length affects the architecture and pattern of innervation differently in leg muscles of mouse, guinea pig, and rabbit compared to those of human and monkey muscles. Anat Rec 262:301-309

Picard B, Lefaucheur L, Berri C, Duclos MJ (2002) Muscle fibre ontogenesis in farm animal species. Reprod Nutr Dev 42:415-431

Quiroz-Rothe E, Rivero JL (2004) Coordinated expression of myosin heavy chains, metabolic enzymes, and morphological features of porcine skeletal muscle fiber types. Microsc Res Tech 65:43-61

Rusnak F, Mertz P (2000) Calcineurin: form and function. Physiol Rev 80:1483-1521

Sacchetto R, Damiani E, Margreth A (2001) Clues to calcineurin function in mammalian fast-twitch muscle. J Muscle Res Cell Motil 22:545-559

Schulz RA, Yutzey KE (2004) Calcineurin signaling and NFAT activation in cardiovascular and skeletal muscle development. Dev Biol 266:1-16

Takahashi M, Kubo T, Mizoguchi A, Carlson CG, Endo K, Ohnishi K (2002) Spontaneous muscle action potentials fail to develop without fetal-type acetylcholine receptors. EMBO Rep 3:674-681

Toniolo L, Patruno M, Maccatrozzo L, Pellegrino MA, Canepari M, Rossi R, D'Antona G, et al. (2004) Fast fibres in a large animal: fibre types, contractile properties and myosin expression in pig skeletal muscles. J Exp Biol 207:1875-1886

Torgan CE, Daniels MP (2006) Calcineurin localization in skeletal muscle offers insights into potential new targets. J Histochem Cytochem 54:119-128

Zhang M, McLennan IS (1998) Primary myotubes preferentially mature into either the fastest or slowest muscle fibers. Dev Dyn 213:147-157

Zhao H, Pestov NB, Korneenko TV, Shakhparonov MI, Modyanov NN (2004) Accumulation of $\beta_{\mathrm{m}}$, a structural member of X,KATPase $\beta$-subunit family, in nuclear envelopes of perinatal myocytes. Am J Physiol Cell Physiol 286:C757-767 